\title{
Diverse molecular forms of plasma B-type natriuretic peptide in heart failure.
}

$\operatorname{AUTHOR}(S)$ :

Nishikimi, Toshio; Minamino, Naoto; Nakao, Kazuwa

\section{CITATION:}

Nishikimi, Toshio ...[et al]. Diverse molecular forms of plasma B-type natriuretic peptide in heart failure.. Current heart failure reports 2011, 8(2): 140-146

ISSUE DATE:

2011-06

URL:

http://hdl.handle.net/2433/142513

\section{RIGHT:}

The final publication is available at www.springerlink.com; This is not the published version. Please cite only the published version.; この論文 は出版社版でありません。引用の際には出版社版をご確認ご利用くだ さい。 


\section{Editorial Manager(tm) for Current Heart Failure Reports} Manuscript Draft

Manuscript Number: HF48

Title: Diverse Molecular Forms of Plasma B-type Natriuretic Peptide in Heart Failure

Article Type: Review Article

Corresponding Author: Toshio Nishikimi, M.D., Ph.D.

Corresponding Author's Institution: Kyoto University Graduate School of Medicine

First Author: Toshio Nishikimi, M.D., Ph.D.

Order of Authors: Toshio Nishikimi, M.D., Ph.D.;Naoto Minamino, Ph.D.;Kazuwa Nakao, M.D., Ph.D. 


\section{Diverse Molecular Forms of Plasma B-type Natriuretic Peptide in Heart Failure}

Running head: Molecular forms of BNP in heart failure

Toshio Nishikimi, $\mathrm{MD}, \mathrm{PhD}^{1)}$, Naoto Minamino, $\mathrm{PhD}^{2)}$, and Kazuwa Nakao, MD, $\mathrm{PhD}^{1)}$

${ }^{1)}$ Department of Medicine and Clinical Science, Kyoto University Graduate School of Medicine, 54, Shogoin-Kawara-cho, Sakyo-ku, Kyoto 606-8507, Japan, and ${ }^{2)}$ Department of Molecular Pharmacology, National Cerebral and Cardiovascular Center Research Institute, Fujishirodai, Suita, Osaka 565-8565, Japan

Total Words counts: 3844 words

Key words: brain natriuretic peptide; atrial natriuretic peptide; heart failure; molecular form; atrial overload; ventricular overload; proBNP-108; BNP-32; processing; secretion; gel-filtration; $O$-glycosylation; natriuretic peptide receptor-A; cGMP,

Address correspondence to:

Toshio Nishikimi, MD, PhD

Department of Medicine and Clinical Science

Kyoto University Graduate School of Medicine

54, Shogoin-Kawara-cho, Sakyo-ku, Kyoto 606-8507, Japan

E-mail: nishikim@kuhp.kyoto-u.ac.jp

TEL: 81-75-751-4287; FAX: 81-75-771-9452 


\begin{abstract}
Recent studies have shown that not only plasma brain natriuretic peptide (BNP)-32, but also plasma proBNP-108 are increased in heart failure (HF) and that current BNP-32 assay kit crossreacts proBNP-108. We also showed that both BNP-32 and proBNP-108 were higher in HF than in normal. The proBNP-108/total BNP (BNP-32+proBNP-108) ratio was widely distributed and HF with ventricular overload had higher proBNP-108/ total BNP ratio than HF with atrial overload. Consistent with this finding, proBNP-108 was the major molecular form in ventricular tissue, and BNP-32 was the major molecular form in atrial tissue. In addition, proBNP-108 was the major molecular form of BNP in pericardial fluid. The proBNP-108/total BNP ratio increased with deterioration of HF and decreased with improvement of HF. Thus, not only BNP-32, but also proBNP-108 is increased in $\mathrm{HF}$ and the proBNP/total BNP ratio also rises in association with pathophysiological conditions such as ventricular overload. A new hypothesis that $O$-glycosylation at $\mathrm{Thr}-71$ in a region close to the cleavage site impairs proBNP-108 processing was proposed. In the future, the precise mechanism of increased proBNP-108 in HF should be elucidated.
\end{abstract}




\section{Introduction}

Atrial natriuretic peptide (ANP) and brain natriuretic peptide (BNP) is a cardiac hormone and a member of the natriuretic family. (1) Both ANP and BNP preferentially bind to natriuretic receptors-A(NPR-A), whereas C-type natriuretic peptide (CNP) specifically binds to NPR-B. The most abundantly expressed in the heart are ANP and BNP, and they are mainly secreted from the cardiac atrium and ventricle, respectively. ANP and BNP play an important role in the regulation of blood pressure and body fluid homeostasis. In addition these peptides have pleiotropic effects, because NPR-A is widely distributed throughout the body (e.g., in the kidneys, vessels, adrenal glands, central nervous system, bone, adipocytes, and heart).

BNP has a strikingly similar to ANP with regard to both its amino acid sequence and its pharmacologic profile (2). Stress to the ventricle such as pressure overload, volume overload, myocardial ischemia, neurohumoral factor and cytokine stimulate BNP gene expression, and proBNP[1-108] (proBNP-108) is produced $(3,4,5)$. Thus, plasma BNP is increased in heart failure and it has been used as a useful biochemical marker for heart failure. When proBNP-108 is secreted from the ventricular myocyte $(4,5)$, it has been thought to be cleaved to proBNP[77-108] (BNP-32) and N-terminal proBNP[1-76] (NT-proBNP-76) in an equimolar fashion by furin, corin or other currently unknown proteases. However, recent studies have shown that not only BNP-32 and NT-proBNP-76, but also proBNP-108 circulates in human plasma and that the proBNP-108level is also increased in heart failure $(6,7,8)$. Other studies have found that the present assay kit for BNP-32 recognizes proBNP-108 at high cross-reactivity in addition to authentic BNP-32 $(9,10)$.

However, the reason why proBNP-108 is secreted without specific conversion into BNP-32 and NT-proBNP-76 remains unknown. The proBNP-108 levels and relative ratio of proBNP-108 to BNP-32 in plasma from patients with heart failure is not well investigated. In addition, plasma proBNP-108 is very 
recently reported to be heavily $O$-glycosylated in its $\mathrm{N}$-terminal peptide $(11,12)$. Thus, it is considered to be important to examine the ratio of proBNP-108 to BNP-32 in the plasma and its relation to pathophysiological conditions of the patients with heart failure.

We review the current status of BNP studies regarding its molecular forms and pathophysiology of heart failure, primarily based on our recent report (13).

\section{Method for the measurement of the proBNP/total BNP ratio in normal and heart failure}

First, we compared plasma BNP-32 and proBNP-108 levels in control subjects, patients with lone atrial fibrillation, and patients with heart failure. Blood samples $(3 \mathrm{~mL})$ were withdrawn from all subjects via the antecubital vein. Blood was immediately transferred into chilled glass tube containing disodium EDTA $(1 \mathrm{mg} / \mathrm{mL})$ and aprotinin $(500 \mathrm{U} / \mathrm{mL})$. Blood was centrifuged immediately at $4^{\circ} \mathrm{C}$ and the plasma was frozen and stored at $-80^{\circ} \mathrm{C}$. Plasma sample was extracted with Sep-Pak C18 cartridges (Waters, Milford, MA, USA) as previously reported method (13). The eluate was lyophilized and dissolved in $30 \%$ acetonitrile containing $0.1 \%$ TFA. An aliquot of the solution was subjected to gel filtration high performance liquid chromatography (HPLC) on a TSK gel G2000SWXL column (7.8 x $300 \mathrm{~mm}$, Tosoh) as previously reported (13). Each fraction was measured by immunofluorescent BNP assay kit (TOSO, Japan). ProBNP-108/total BNP ratio was calculated based on the summation of a high molecular weight (MW) immunoreactive (IR-) proBNP-108 and a low MW IR-BNP-32 with the following formula: proBNP-108/total BNP ratio = IR-proBNP-108/(IR-proBNP-108+IR-BNP-32).

Since very recent studies have revealed that glycosylated pro-BNP of MW about $35 \mathrm{~K}$ is circulating in plasma $(11,12)$, we examined their elution positions of recombinant proBNP-108 (HyTest, Finland), glycosylated proBNP-108 (HyTest, Finland), and synthetic BNP-32 (Peptide Institute, Japan) in the gel filtration. To 
evaluate the cross-reactivity of proBNP-108 and glycosylated proBNP-108 in the immunofluorescent assay (FIA) kit for BNP-32, an aliquot of each peptide was subjected to acid hydrolysis at $110 \mathrm{C}$ for $22 \mathrm{~h}$ to estimate peptide contents by amino acid analysis (L-8500 analyzer, Hitachi, Tokyo, Japan), and another aliquot was submitted to the FIA for BNP-32, after desalting purchased peptides. In this system, we were able to accurately evaluate the proBNP/total BNP ratio because we used one assay system almost equivalently recognizing BNP-32 and proBNP-108. Some studies evaluated the proBNP/total BNP ratio by using a distinct assay system for proBNP or BNP-32. In such a study, different affinities of antibodies against proBNP, BNP-32 and their related peptides in each system hamper accurate evaluation of the proBNP/total BNP ratio.

\section{Plasma concentrations of IR-BNP-32, IR-proBNP-108 and proBNP/total BNP ratios in control, atrial fibrillation, and heart failure.}

As shown in Figures 1-A and 1-B, two peaks of IR-BNP were constantly observed in all cases. The first peak was observed in fractions \#9-16 of MW larger than $13 \mathrm{~K}$, and the second peak was in fractions \#18-21 of MW 3.5K corresponding to BNP-32. In this gel filtration HPLC, recombinant proBNP-108 and glycosylated proBNP-108 were eluated mainly in fraction \#14 and fraction \#15, being not separable of glycosylated proBNP-108 and non-glycosylated proBNP-108 with each other. In the following part the term IR-proBNP-108 indicates high MW IR-BNP, which includes both proBNP-108 and glycosylated proBNP-108, while IR-BNP-32 indicates low MW IR-BNP that is mainly composed of BNP-32. The proBNP-108/total BNP ratio was narrowly distributed in control and atrial fibrillation; however, the proBNP-108/total BNP ratio was widely distributed in patients with heart failure (Figure 1-C). Thus, the mean proBNP-108/total BNP ratio in heart failure was significantly lower in heart failure than in control or atrial fibrillation.

It is thought that proBNP-108 is cleaved to BNP-32 and N-terminal 
proBNP-76 in an equimolar fashion by a processing enzyme, when proBNP-108 is secreted from the ventricular myocyte $(1,5)$. However, recent studies have shown that not only BNP-32, but also proBNP-108 circulates in human plasma and they are increased in patients with heart failure (7-9). However, there were few studies, which measured both BNP-32 and proBNP-108 using the same assay kit. From these results, we confirmed that high and low MW forms of BNP, BNP-32 and proBNP-108, were present in plasma from control subjects and patients with atrial fibrillation and heart failure, suggesting that a significant percentage of proBNP-108 is secreted from the heart without processing.

\section{Comparison of plasma ProBNP/total BNP ratio of ventricular overload with that of atrial overload}

Next, we measured plasma BNP-32 and proBNP-108 in patients with heart failure with atrial overload and heart failure with ventricular overload. Heart failure with atrial overload included mitral stenosis, mitral regurgitation, and atrial septal defect, whereas heart failure with ventricular overload included aortic regurgitation and aortic stenosis. We compared the ratio of proBNP-108/total BNP in heart failure with atrial overload to that in heart failure with ventricular overload. Two peaks of IR-BNP were observed in both groups. Interestingly, IR-BNP-32 peak was more dominant than IR-proBNP-108 peak in heart failure with atrial overload; in contrast, IR-proBNP-108 and IR-BNP-32 peaks were nearly equivalent in heart failure with ventricular overload. As a result, the mean proBNP-108/total BNP ratio was higher in heart failure with ventricular overload than that in heart failure with atrial overload (Figure 2-A). These results suggest that ventricular overload alters processing of proBNP-108 (13).

\section{Tissue concentrations of proBNP and BNP-32 in ventricle and atrium}

To elucidate the mechanism for the higher proBNP-108/total BNP ratio in ventricular overload than atrial overload, we analyzed molecular forms of BNP in 
ventricular and atrial tissues. Resected samples of left atrial tissues and left ventricles were frozen in liquid nitrogen, and stored at $-80^{\circ} \mathrm{C}$. The atrial and ventricular tissues were boiled in 10 volumes of $1 \mathrm{~mol} / \mathrm{L}$ acetic acid to inhibit the endogenous peptidase activity (14). The tissues were homogenized, centrifuged, lyophilized, and subjected to gel filtration HPLC on a TSK gel G2000SWXL column as described above for plasma. Then, each fraction was submitted to the FIA for BNP-32 similar to the plasma samples. Two IR-BNP peaks corresponding to BNP-32 and proBNP-108 were also observed in atrial and ventricular tissue. Interestingly, in atrial tissue, the low MW IR-BNP peak corresponding to BNP-32 was a dominant molecular form of IR-BNP; in contrast, the high MW IR-BNP peak, corresponding to proBNP-108 was a dominant molecular form in ventricular tissue. Consequently, the mean proBNP-108/total BNP ratio was much higher in ventricular tissue than in atrial tissue (Figure 2-B).

There were few studies, which measured molecular forms of BNP in cardiac tissue. A previous study showed that two molecular forms of IR-BNP of MW 4K and MW 13-15K were present in human atrial tissue (15), which is consistent with the present results. However, to our best of knowledge, no study has previously examined the molecular form of BNP in human ventricular tissue. Although the exact meaning of dominant presence of proBNP-108 in ventricular tissue remains unknown at present, these results suggest that alteration of proBNP processing may occur when some stress is loaded onto ventricle.

\section{Pericardial fluid concentrations of IR-BNP-32 and IR-proBNP-108 in patients with heart failure}

To confirm the molecular form of BNP produced and secreted from the ventricular tissue, we characterized IR-BNP-32 and IR-proBNP-108 in pericardial fluid and plasma in patients who underwent cardiac surgery. Two IR-BNP peaks corresponding to BNP-32 and proBNP-108 in plasma and pericardial fluid in both patients with mitral regurgitation and with aortic stenosis 
were present. In plasma, IR-BNP-32 was a dominant molecular form in patients with mitral regurgitation, while IR-proBNP-108 was a dominant molecular form in patients with aortic stenosis; however, IR-proBNP-108 was exclusively the dominant molecular form in pericardial fluid in both cases. Consequently, the mean proBNP-108/total BNP ratio was greater in pericardial fluid than in the plasma (Figure 2-C).

Pericardial fluid is known to contain abundant levels of various bioactive substances produced in the heart $(16,17)$ and its composition is known to be close to the interstitial fluid in the ventricle (18). In addition, the concentrations of bioactive substances, such as adrenomedullin, BNP, ANP, basic fibroblast growth factor, and vascular endothelial growth factor, have been reported to be higher in pericardial fluid than in plasma (16-19). Most IR-BNP was present as IR-proBNP-108, irrespective of the type of heart failure. These results support the hypothesis again that proBNP-108 is the major molecular form of BNP in the ventricle and that most of proBNP-108 is secreted from ventricle without proteolytic processing.

\section{The post-treatment or natural course of plasma concentrations of IR-BNP-32 and IR-proBNP-108 in patients with heart failure}

To investigate whether the pathophysiological status of heart failure affects the molecular form of BNP in plasma, plasma proBNP-108 and BNP-32 levels were measured in patients with heart failure before and after their symptom had improved in response to treatments. These peptides levels were also measured in patients with heart failure before and after their symptom had deteriorated. Elevated plasma IR-BNP levels decreased after the treatments, accompanied by a reduction in the proBNP-108/total BNP ratio. In the cases that heart failure deteriorated during the observation, IR-BNP levels increased concomitantly with an increase in the proBNP-108/total BNP ratio.

Thus, it is concluded that the proBNP-108/total BNP ratio is altered 
depending on the pathophysiological status of heart failure. An increased proBNP-108/total BNP ratio in severe heart failure may be explained in part by the increased production and secretion of proBNP-108 from the ventricle. Another possibility is that gene expression of proteolytic processing enzyme is not increased in parallel with the increase in gene expression of BNP precursor in severe heart failure, which results in the reduced proteolytic conversion of proBNP-108 into BNP-32.

\section{The pathophysiological significance of increased proBNP in heart failure}

In vitro studies have shown remarkably reduced cGMP producing ability of proBNP-108 in vascular smooth muscle and endothelial cells compared with that of BNP-32 [11]. The plasma cGMP level is increased in proportion to the severity of mild to moderate HF, and levels of plasma cGMP correlates with those of plasma BNP. However, increased cGMP levels are attenuated in severe heart failure [20], of which mechanism has not been fully elucidated. A definite increase in the amount of less hormonally active proBNP-108 observed in severe heart failure might explain this phenomenon. Indeed, the proBNP-108/BNP-32 ratio is increased in decompensated heart failure and that medical therapy for such patients decreases plasma BNP and patients' symptoms in concert with a reduction in the proBNP-108/BNP-32 ratio [13]. Therefore, elucidation of the mechanism underlying the increased ratio of proBNP-108/BNP-32 should help to clarify the pathogenesis of heart failure and/or pave the way towards novel therapies.

\section{The mechanism of proBNP secretion without processing: new hypothesis of processing of proBNP}

Like the increasing levels of proBNP-108, BNP-32 and NT-proBNP-76 in heart failure, proBNP-108 is also $O$-glycosylated according to heart failure severity $[12,22]$. Thus, understanding the clinical relevance of proBNP-108 
glycosylation is a matter of obvious importance.

Pressure overload, volume overload, ischemia and other conditions stimulate BNP gene transcription. The BNP mRNA is translated in the endoplasmic reticulum to produce preproBNP. Removal of its signal peptide generates proBNP-108 that can be posttranslationally glycosylated to varying degrees at several sites (Ser36, Thr37, Thr44, Thr48, Thr53, Ser58, and Thr71) of its $\mathrm{N}$-terminal region throughout the Golgi network [22]. O-glycosylated (glyco-) proBNP-108 is transported to the trans-Golgi network, where it is cleaved to BNP-32 and NT-proBNP-76 probably by furin [22,23]. Both BNP-32 and NT-proBNP-76 are thought to be secreted via a constitutive pathway without storing in secretory granules (Figure 3).

Levels of glyco-proBNP-108, but not of proBNP-108, are increased in the plasma of patients with severe heart failure [24,25]. Western blot analyses have revealed the presence of high MW (20-22 K) IR-BNP and low MW (4 K) IR-BNP species in the plasma of such patients. Digestion with deglycosylation enzymes for $8 \mathrm{~h}$ reduced the 20-22K BNP species to about $12 \mathrm{~K}$, which is the predicted size of pro-BNP-108, whereas the $4 \mathrm{~K}$ form, corresponding to BNP-32, remained unchanged [11]. Thus, glyco-proBNP-108 definitely circulates in the plasma of patients with heart failure and is a major molecular form of proBNP.

How glyco-proBNP-108 is secreted without processing under conditions of severe heart failure is not fully understood. A recent study has shown that $O$-glycosylation at $\mathrm{Thr} 71$ in a region close to the cleavage site impairs proBNP-108 processing by furin in HEK293 cell, a specific cell line originally derived from human embryonic kidney cells [22,23] (Figure 3). Since the effect of $O$-glycosylation was only evaluated with furin in that vitro study, the roles of other possible processing enzymes, such as corin, remains unclear. It has not been examined whether this finding is also valid in the in the cardiac myocytes of atrium and ventricle in vitro, either. Therefore, further studies using cardiac myocytes are required to elucidate the exact mechanism of proBNP-108 
processing and the effect of $O$-glycosylation.

\section{Conclusion}

We review the recent studies regarding complex molecular forms of BNP, in plasma and tissue, primarily based on our recent results. The presence of proBNP-108 in plasma in patients with heart failure has almost been established. Recent studies also showed that the proBNP/total BNP ratio increases in association with pathophysiological conditions such as ventricular overload. An interesting hypothesis that $O$-glycosylation at $\mathrm{Thr} 71$ in a region close to the cleavage site impairs proBNP-108 processing by furin is proposed based on the experiments in HEK293 cell (Figure 3). Whether this hypothesis is true in cardiac myocytes should be investigated in the near future. 


\section{ACKNOWLEDGMENTS}

We thank Dr. Masashi Ikeda for helpful advice. We thank Mr. Kazumi Akimoto, Ms. Masako Matsubara, Ms. Keiko Ishikawa, Ms. Masako Minato, Ms. Kyoko Tabei, and Ms. Machiko Sakata for technical assistance.

Funding: This work was supported in part by Scientific Research Grants-in-Aid ['18590787' and '20590837'] from the Ministry of Education, Culture, Sports, Science and Technology of Japan, by the Fund from the Suzuken Memorial Foundation, by the Science Research Promotion Fund from the Promotion and Mutual Aid Corporation for Private Schools of Japan; and by the Intramural Research Fund of National Cerebral and Cardiovascular Center of Japan.

Competing interests: None. 


\section{FIGURE LEGENDS}

Figure 1: ProBNP-108/total BNP ratios in plasma in the control, atrial fibrillation and heart failure.

(A, B) Gel filtration HPLC profile of the venous plasma extracts from control (A) and heart failure (B). Two peaks of high MW and low MW IR-BNPs corresponding to proBNP-108 and BNP-32 were observed. (C) The proBNP-108/total BNP ratios in plasma in the control, atrial fibrillation (Af) and heart failure (HF). (modified Ref. 13)

Figure 2: ProBNP-108/total BNP ratios in plasma in heart failure patients with atrial overload and ventricular overload, cardiac tissue, and pericardial fluid

(A) The proBNP-108/total BNP ratio in heart failure patients with atrial overload and those with ventricular overload. (B) The proBNP-108/total BNP ratio in the atrial and ventricular tissue in patients with heart failure who underwent cardiac surgery. (C) The proBNP-108/total BNP ratios in pericardial fluid and plasma from the same patients who underwent cardiac surgery. (modified Ref. 13)

Figure 3. Schematic representation of processing and glycosylation of proBNP in cardiomyocytes.

Some of the biosynthesized proBNP-108 is $O$-glycosylated in Golgi apparatus. ProBNP-108 is converted into BNP-32 and NT-proBNP-76 by processing enzyme in trans-Golgi network, if $O$-glycosylation does not occur at Thr71. Then, BNP-32 and NT-proBNP-76 is secreted in an equimolar fashion via a constitutive pathway. If proBNP is $O$-glycosylated at $\mathrm{Thr} 71$ and is not cleaved by processing enzyme, non-cleaved glycosylated proBNP-108 is secreted into circulation. 


\section{REFERENCES}

1. Minamino N, Horio H, Nishikimi T: Chapter 165. Natriuretic peptides in the cardiovascular system. In: Kastin AJ, ed. THE HANDBOOK OF BIOLOGICALLY ACTIVE PEPTIDES. Academic Press, 2006. P. 1217-1225

2. Sudoh T, Kangawa K, Minamino N, Matsuo H: A new natriuretic peptide in porcine brain. Nature. 1988;332:78-81.

3. Nishikimi T, Maeda N, Matsuoka $\mathrm{H}$ : The role of natriuretic peptides in cardioprotection. Cardiovasc Res. 2006;69:318-28.

4. Daniels LB, Maisel AS: Natriuretic peptides. J Am Coll Cardiol. 2007;50:2357-68.

5. Weber M, Hamm C: Role of B-type natriuretic peptide (BNP) and NT-proBNP in clinical routine. Heart. 2006;92:843-9.

6. Lam CS, Burnett JC, Jr., Costello-Boerrigter L, et al.: Alternate circulating pro-B-type natriuretic peptide and B-type natriuretic peptide forms in the general population. J Am Coll Cardiol. 2007;49:1193-202

7. Waldo SW, Beede J, Isakson S, et al.: Pro-B-type natriuretic peptide levels in acute decompensated heart failure. J Am Coll Cardiol. 2008;51:1874-82.

8. Seferian KR, Tamm NN, Semenov AG, et al.: The brain natriuretic peptide (BNP) precursor is the major immunoreactive form of BNP in patients with heart failure. Clin Chem. 2007;53:866-73

9. Shimizu H, Masuta K, Asada H, et al.: Characterization of molecular forms of probrain natriuretic peptide in human plasma. Clin Chim Acta. 2003;334:233-9. 10. Nishikimi T, Minamino N, Horii K, Matsuoka H: Do commercially available assay kits for B-type natriuretic peptide measure Pro-BNP1-108, as well as BNP1-32? Hypertension. 2007;50:e163

11. Liang F, O'Rear J, Schellenberger U, et al.: Evidence for functional heterogeneity of circulating B-type natriuretic peptide. J Am Coll Cardiol. 2007;49:1071-8.

12. Schellenberger U, O'Rear J, Guzzetta A, et al.: The precursor to B-type 
natriuretic peptide is an O-linked glycoprotein. Arch Biochem Biophys. 2006;451:160-6.

13. **Nishikimi T, Minamino N, Masashi I, et al.: Diversity of Molecular Forms of Plasma Brain Natriuretic Peptide in Heart failure -Different proBNP-108 to BNP-32 Ratios in Atrial and Ventricular Overload- Heart. 2010; 96:432-439 This study first shows the relation between increase of proBNP-108 and ventricular overload in patients with heart failure.

14. Yoshihara F, Nishikimi T, Sasako Y, et al.: Plasma atrial natriuretic peptide concentration inversely correlates with left atrial collagen volume fraction in patients with atrial fibrillation: plasma ANP as a possible biochemical marker to predict the outcome of the maze procedure. J Am Coll Cardiol. 2002;39:288-94. 15. Hino J, Tateyama H, Minamino N, et al.: Isolation and identification of human brain natriuretic peptides in cardiac atrium. Biochem Biophys Res Commun. 1990; 167:693-700.

16. Nishikimi T, Shibasaki I, Iida H, et al.: Molecular forms of adrenomedullin in pericardial fluid and plasma in patients with ischaemic heart disease. Clin Sci (Lond). 2002;102:669-77.

17. Nishikimi T, Asakawa H, Iida $H$, et al.: Different secretion patterns of two molecular forms of cardiac adrenomedullin in pressure- and volume-overloaded human heart failure. J Card Fail. 2004;10:321-7.

18. Fujita M, Ikemoto M, Kishishita M, et al.: Elevated basic fibroblast growth factor in pericardial fluid of patients with unstable angina. Circulation. 1996;94:610-3.

19. Tambara K, Fujita M, Nagaya N, et al.: Increased pericardial fluid concentrations of the mature form of adrenomedullin in patients with cardiac remodelling. Heart. 2002;87:242-6.

20. Tsutamoto T, Wada A, Maeda K, et al.: Attenuation of compensation of endogenous cardiac natriuretic peptide system in chronic heart failure: prognostic role of plasma brain natriuretic peptide concentration in patients with chronic 
symptomatic left ventricular dysfunction. Circulation. 1997;96:509-16.

21. Hammerer-Lercher A, Halfinger B, Sarg B, et al.: Analysis of circulating forms of proBNP and NT-proBNP in patients with severe heart failure. Clin Chem. 2008;54:858-865.

22. **Semenov AG, Postnikov AB, Tamm NN, et al.: Processing of pro-Brain natriuretic peptide is suppressed by $\mathrm{O}$-glycosylation in the region close to the cleavage site. Clin Chem. 2009;55:493-498. This study first shows the O-glycosylation at Thr-71 in a region close to the cleavage site impairs proBNP-108 processing by furin in HEK293 cell.

23. *Semenov AG, Tamm NN, Seferian KR, et al.: Processing of pro-B-type natriuretic peptide: furin and corin as candidate convertases. Clin Chem. 2010;56:1166-1176. This study shows that both furin and corin are involved in the proBNP processing pathway.

24. Luckenbill KN, Christenson RH, Jaffe AS, et al.: Cross-reactivity of BNP, NT-proBNP, and proBNP in commercial BNP and NT-proBNP assays: preliminary observations from the IFCC Committee for Standardization of Markers of Cardiac Damage. Clin Chem. 2008;54:619-621

25. Seferian KR, Tamm NN, Semenov AG, et al.: Immunodetection of glycosylated NT-proBNP circulating in human blood. Clin Chem. 2008;54:866-873. 

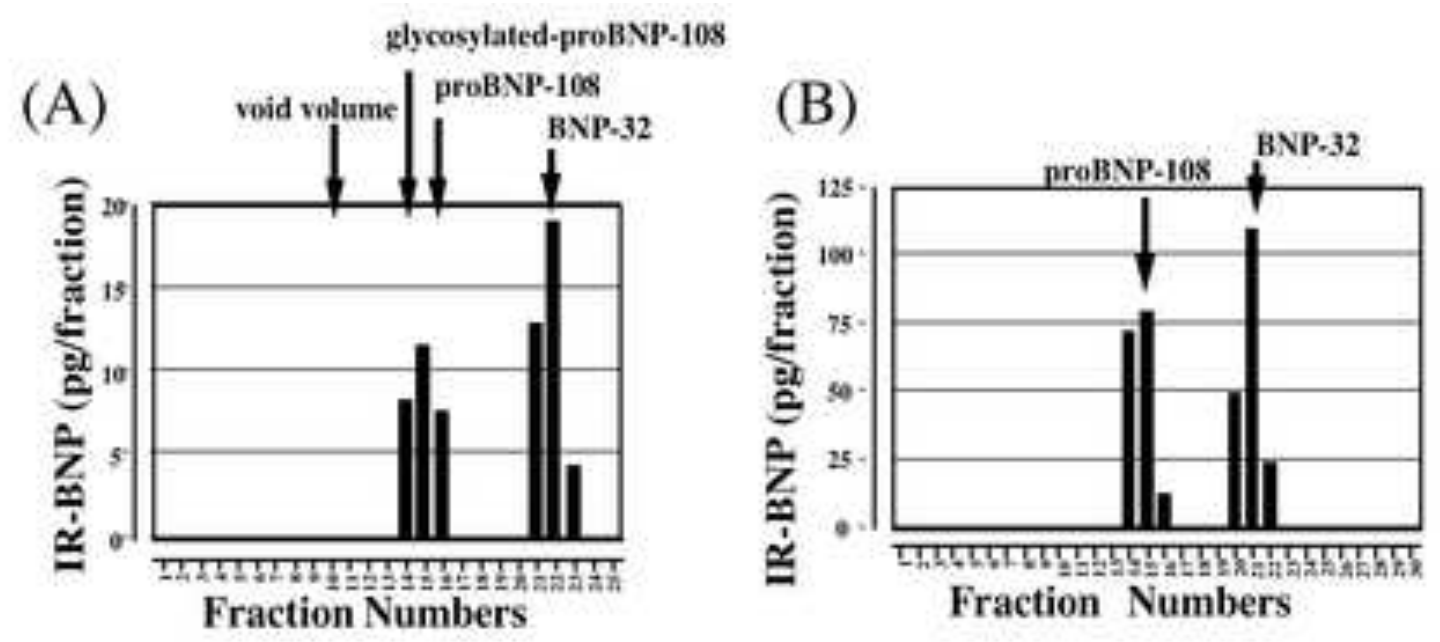

(C)

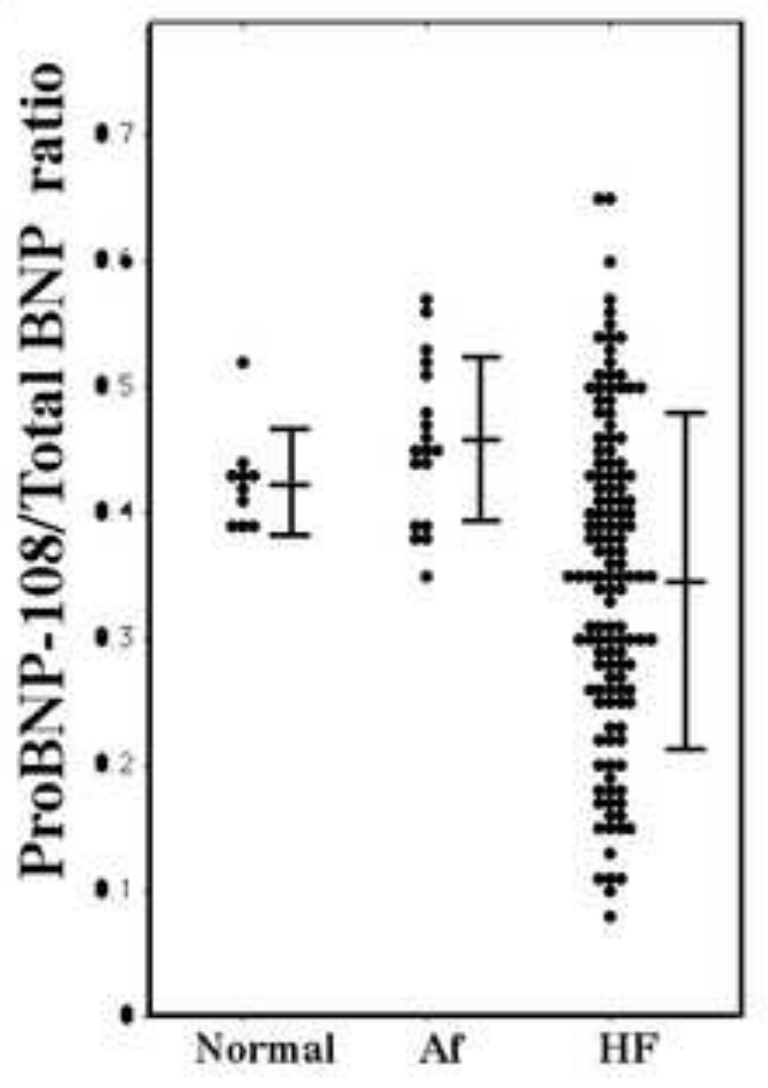


(A)

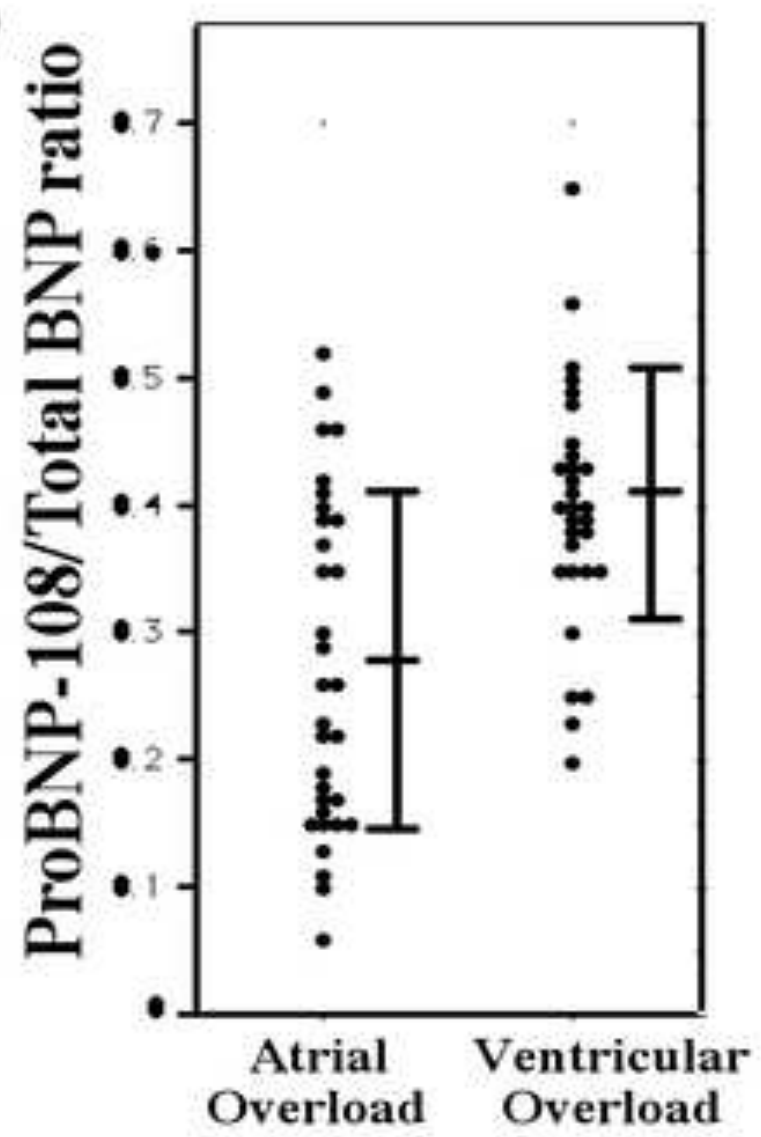

(B)

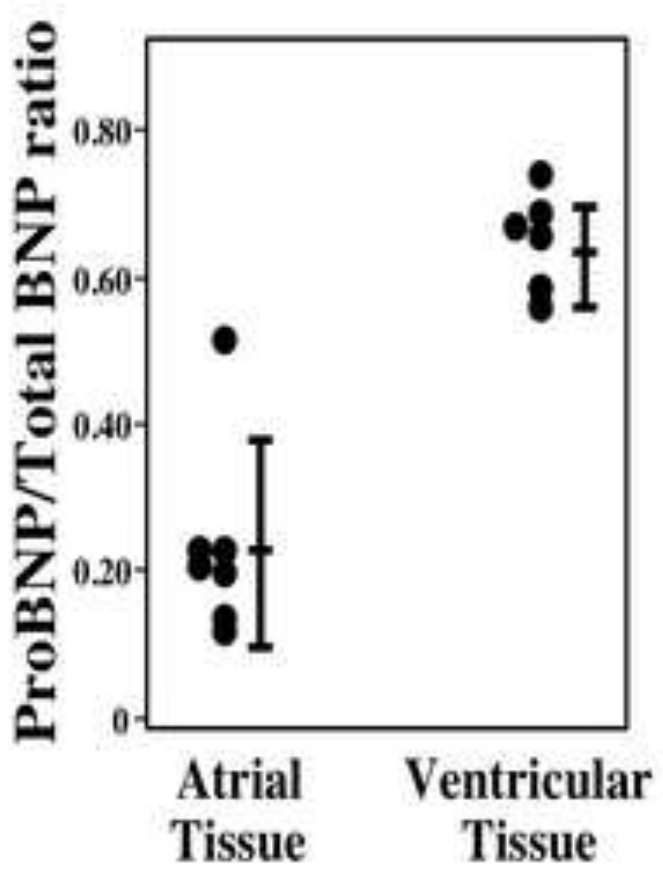

(C)

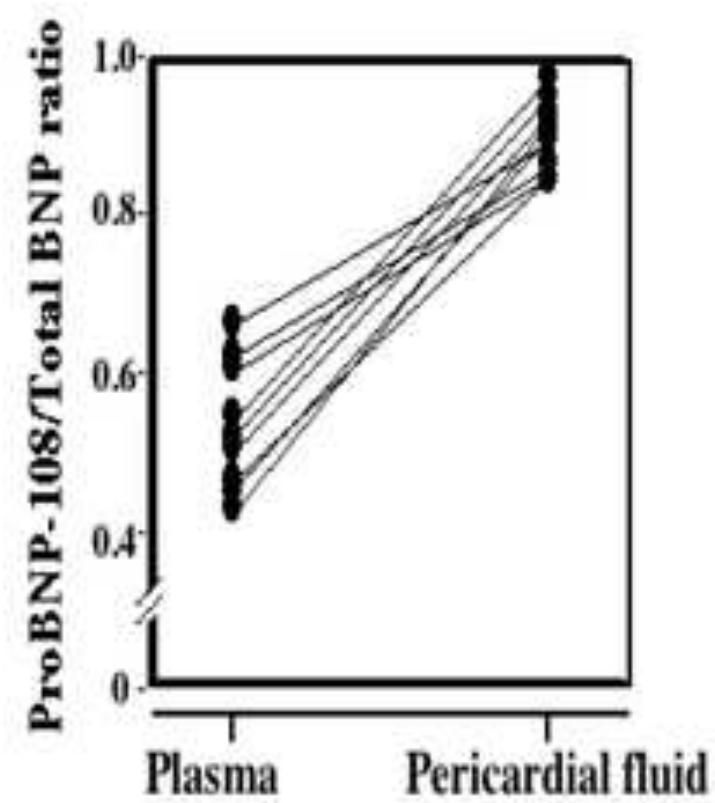




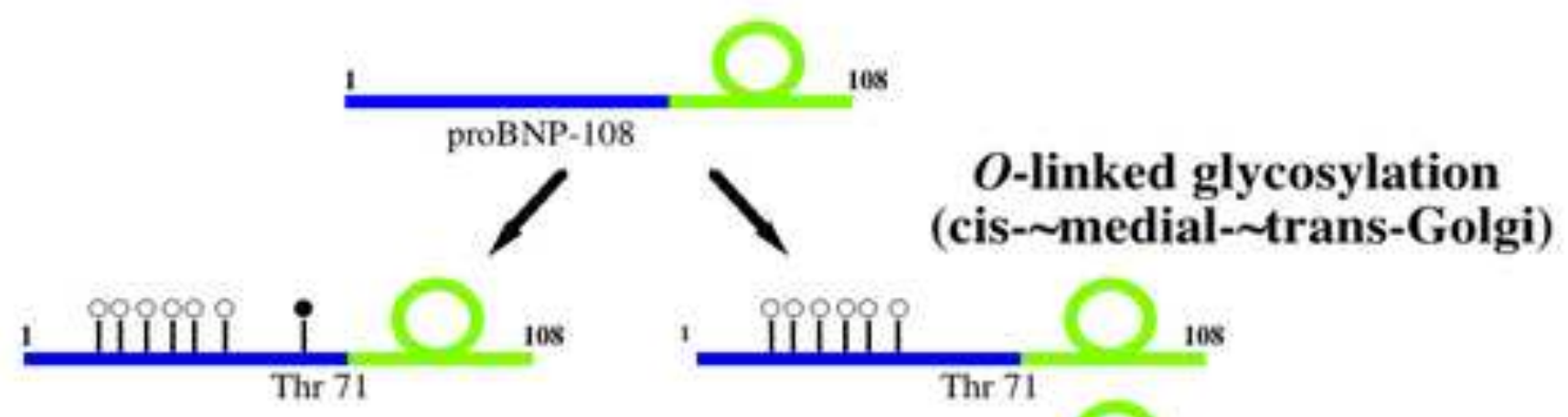

not cleaved by processing enzyme

(trans-Golgi network)

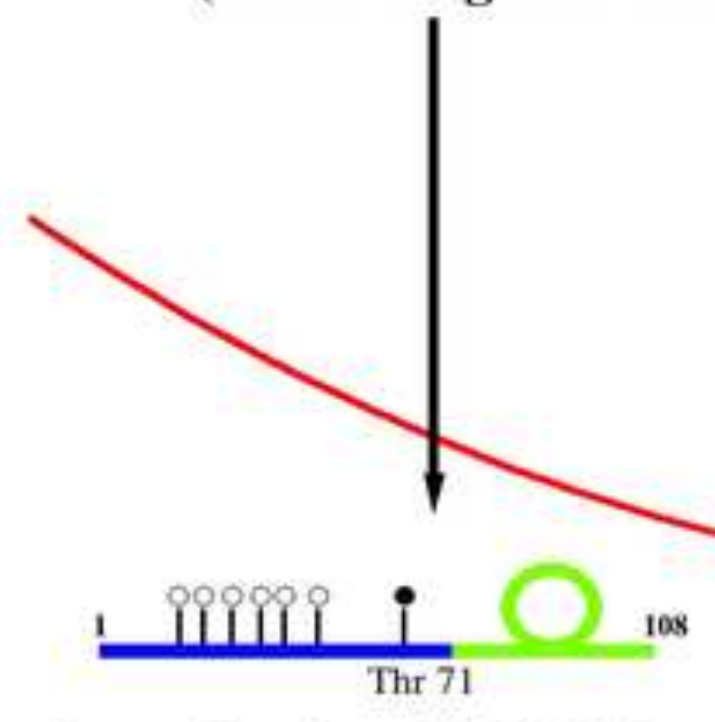

glycosylated proBNP-108

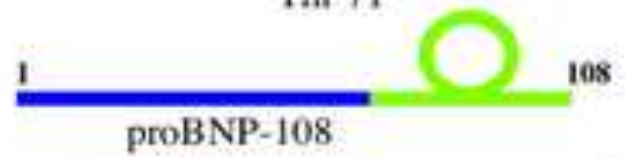

cleaved by processing enzyme
(trans-Golgi network)

(trans-Golgi network) Thr 71

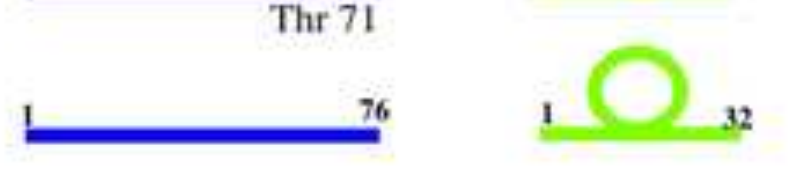

10001

Thr 71

glycosylated N-terminal proBNP-76

BNP-32 